\title{
Universiteit
}

Leiden

The Netherlands

\section{Orthogonal fields: A path to long-range three-dimensional order in block copolymers}

Zvelindovsky, A.V.; Sevink, G.J.A.

\section{Citation}

Zvelindovsky, A. V., \& Sevink, G. J. A. (2005). Orthogonal fields: A path to long-range threedimensional order in block copolymers. Journal Of Chemical Physics, 123(7), 074903. doi:10.1063/1.2000231

Version: $\quad$ Not Applicable (or Unknown)

License: $\quad$ Leiden University Non-exclusive license

Downloaded from: https://hdl.handle.net/1887/68052

Note: To cite this publication please use the final published version (if applicable). 
Orthogonal fields: A path to long-range three-dimensional order in block copolymers

A. V. Zvelindovsky, and G. J. A. Sevink

Citation: The Journal of Chemical Physics 123, 074903 (2005); doi: 10.1063/1.2000231

View online: https://doi.org/10.1063/1.2000231

View Table of Contents: http://aip.scitation.org/toc/jcp/123/7

Published by the American Institute of Physics

\section{Articles you may be interested in}

Long-range ordered structures in diblock copolymer melts induced by combined external fields

The Journal of Chemical Physics 121, 1609 (2004); 10.1063/1.1763140

Block Copolymers-Designer Soft Materials

Physics Today 52, 32 (1999); 10.1063/1.882522

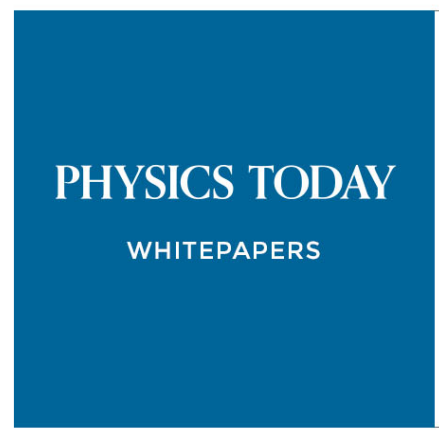




\title{
Orthogonal fields: A path to long-range three-dimensional order in block copolymers
}

\author{
A. V. Zvelindovsky \\ Materials Science Centre, Department of Physics, Astronomy and Mathematics, \\ University of Central Lancashire, Preston PR1 2HE, United Kingdom \\ G. J. A. Sevink \\ Leiden Institute of Chemistry, Leiden University, P.O. Box 9502, 2300 RA Leiden, The Netherlands
}

(Received 19 May 2005; accepted 20 June 2005; published online 22 August 2005)

Large-scale computer simulations show that two orthogonal external fields can control the orientation of lamellar microdomains in diblock copolymers in three dimensions and lead to an enhanced long-range ordering. () 2005 American Institute of Physics. [DOI: 10.1063/1.2000231]

\section{INTRODUCTION}

Long-range ordering of microdomains in block copolymers is a requisite for applications that require spatial definition of structures in three dimensions. Thin films of block copolymers with microdomains oriented normal to the surface are increasingly used as templates and scaffolds for functional nanoscopic materials. ${ }^{1}$ Many methods have been developed, such as simple shear and elongational flow fields, electric fields, surface fields, and solvent flow, to achieve the desired microdomain orientation. ${ }^{2-9}$ Recently, $\mathrm{Xu}$ et al. in a set of elegant experiments have shown that the application of sequential orthogonal flow and electric fields can lead to an excellent long-range three-dimensional (3D) order and a single orientation of lamellar microdomains. ${ }^{10}$ Each of the fields alone is not enough to facilitate such a high degree of perfection, and is, in particular, in the case of thin films, often not able to give a desired orientation at all. In the present paper we investigate this experimental phenomenon by means of large-scale dynamic self-consistent-field simulations. The systematic variation of field compositions studied suggests that a field composition, different from the one used in Ref. 10, can result in an even higher degree of ordering. The first simulation of the effect of orthogonal shear and electric field for a two-dimensional (2D) lamellar system has been reported earlier in Ref. 11. The subject of the present paper is essentially $3 \mathrm{D}$ ordering.

One way to produce well-aligned microdomains textures by flow fields is, for example, a roll-casting process, which has proven to be successful in bulk samples. ${ }^{12,13}$ Shear flow can promote different orientations of microdomains, depending on the interplay of several factors such as shear rate, viscosity, and temperature. ${ }^{14}$ Due to the nature of the flow, which is always affected by the various boundaries present in the system, the resulting microstructure will have defects in the ordering. However, if one wants to use block copolymer materials as templates in nanoelectronics, the density of structural defects should be significantly low, and eventually zero. A different type of external field used for microstructure orientation is an electric field. In thin films, electric fields have been effectively used to achieve orientation of the microdomains normal to the surface. ${ }^{15,16}$ However, such fields are unidirectional, causing a high degree of orientation along the field lines, but lacking any preferred orientation in the plane normal to this direction. Consequently, a second field, orthogonal to this field, is required to achieve morphologies where the microdomain orientation is controlled in all three dimensions.

Here, we show that a combination of shear flow and an orthogonal electric field produces an excellent 3D ordering in a lamellae-forming diblock copolymer melt. The suggestion that a combination of weak shear flow and an electric field might lead to interesting results for a lamellar system can already be found in Ref. 17, albeit as a remark without theoretical analysis.

\section{RESULTS AND DISCUSSION}

We employ the dynamic version of the self-consistentfield theory, which describes the dynamics of microphase separation in block copolymers (modeled as Gaussian chains in the mean field of all other molecules). ${ }^{18}$ The phase separation can be monitored by the scalar $\psi_{I}(\mathbf{r}, t)$, which is the deviation of the concentration of a polymer block $I$ from its average value. In the case of an incompressible diblock copolymer melt the system is described by only a single $\psi$. The time evolution of $\psi$ in the simplest case follows a diffusiontype equation, ${ }^{19}$

$$
\dot{\psi}=M \nabla^{2} \mu+\eta,
$$

with a constant mobility $M$, chemical potential $\mu$, thermal noise $\eta$, and a proper choice of the boundary conditions. In the presence of simple shear flow with velocity $\nu_{x}=\dot{\gamma} z, \nu_{y}$ $=\nu_{z}=0$ one can replace Eq. (1) by a diffusion-convection equation, ${ }^{20}$

$$
\dot{\psi}=M \nabla^{2} \mu-\dot{\gamma} z \nabla_{x} \psi+\eta,
$$

where $\dot{\gamma}$ is a constant shear rate. The chemical potential in the presence of an electric field $\mathbf{E}$ has the form $\mu=\mu^{0}$ $-(\partial \varepsilon / \partial \psi)_{T} E^{2} / 8 \pi{ }^{21}$ where $\mu^{0}$ is the chemical potential in the absence of the electric field, and $\varepsilon$ is the dielectric constant of the polymeric material, which can be approximated as $\varepsilon \approx \varepsilon_{c}+\varepsilon_{1} \psi{ }^{19}$ Notice that, as $\psi$ has the dimension of inverse 


\section{Shear rate}

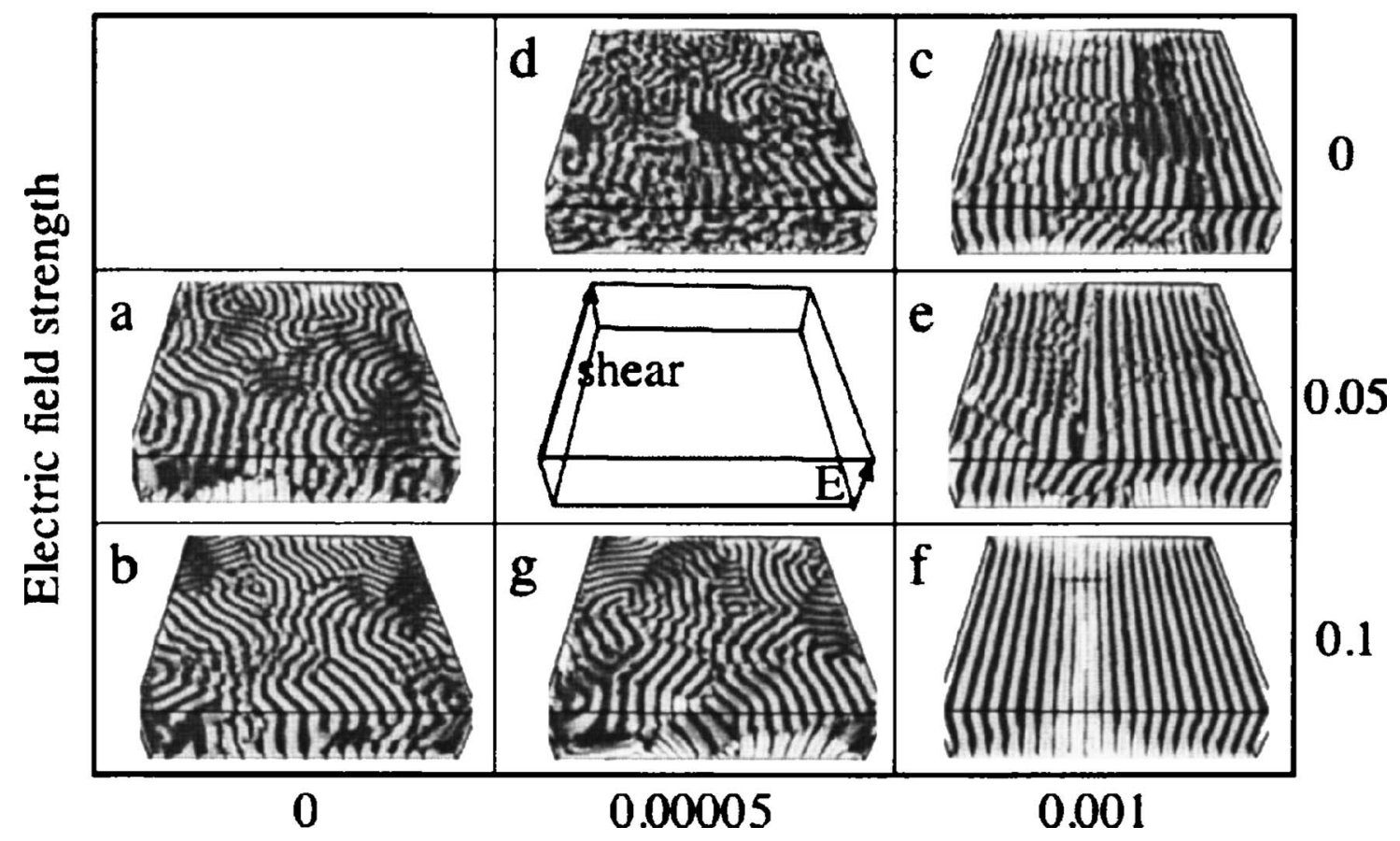

FIG. 1. Diagram of the simulation snapshots of the diblock copolymer melt in single and combined external fields (all at the same time stages after 4000 time steps). The considered dimensionless values of $\widetilde{\alpha}$ and $\tilde{\dot{\gamma}}$ are denoted on the axes.

volume, $\varepsilon_{c}$ and $\varepsilon_{1}$ are of different dimensions. We choose the applied electric field to be orthogonal to the shear plane in Eq. (2), $\mathbf{E}_{0}=\left(0,0, E_{0}\right)$. The electric field inside the material $\mathbf{E}$ deviates from the applied electric field $\mathbf{E}_{0}$, satisfying the Maxwell equation $\operatorname{div} \varepsilon \mathbf{E}=0$, where $\mathbf{E}=\mathbf{E}_{0}-\nabla \varphi$. Keeping only the leading terms, one can rewrite Eq. (2) in the form $^{11,22}$

$$
\dot{\psi}=M \nabla^{2} \mu^{0}+\alpha \nabla_{z}^{2} \psi-\dot{\gamma} z \nabla_{x} \psi+\eta,
$$

where $\alpha \equiv M\left(\varepsilon_{1} E_{0}\right)^{2} / 4 \pi \varepsilon_{c}$. The chemical potential without the electrostatic contribution $\mu^{0}$ is calculated in a standard way using the self-consistent field theory for the ideal Gaussian chains, with the mean-field interactions between copolymer blocks $A$ and $B$ described by a parameter $\varepsilon_{A B}{ }^{18}$

The model system we study in the following is a symmetric $A_{4} B_{4}$-copolymer melt with the mean-field interactions $\varepsilon_{A B}=7 \mathrm{~kJ} / \mathrm{mol}(\chi N=22.4)$. The simulations have been performed in a large 3D box $x \times y \times z=128 \times 128 \times 32$, starting with a homogeneous mixture. The large scale of the simulation is crucial for the phenomenon under investigation, because of the influence of the periodic (or sheared periodic) boundary conditions on the simulated structure. This boundary condition can induce artificial ordering, especially for (too) small box sizes. As our goal is to investigate defects in the system, the artificial "energy" of boundary conditions should be small compared to the energy of an individual defect in the microstructure. It implies that the size of the simulation box should be sufficiently large when compared to the characteristic size of a typical lamellar grain. The main objective in this type of research is to reach a lateral long-range ordering, which is desirable from a technological point of view. To this purpose it is sufficient to choose the simulation box large only in lateral $(x, y)$ directions. We are not aiming to mimic any specific chemical system, therefore, all simulations have been performed in dimensionless units with the shear flow strength and the electric-field strength being parametrized by $\tilde{\dot{\gamma}} \equiv \dot{\gamma} h^{2} / 2 k T M \nu_{p}, \widetilde{\alpha} \equiv \alpha / k T M \nu_{p}$, respectively (in which $\nu_{p}$ is the volume of the polymer chain and $h$ is the grid mesh size ${ }^{18,20}$ ).

Figures 1(a) and 1(b) show snapshots of the simulation of the diblock copolymer melt after the application of electric field only. The presence of multiple grains in our simulation is quite remarkable, when compared to experimental results for an applied electric field only, in which case many grains are actually found. ${ }^{23}$ The presence of these grains can be explained by the electric field having only one preferred direction, namely, the field direction. While the lamellae are perfectly oriented parallel to the direction in which the electric field is applied ( $z$ axis), there is no force associated with the electric field, which would be able to rotate different grains in the lateral $x-y$ plane. The only force present to promote the formation of one single large grain is due to the energy cost of grain boundaries, which is not sufficiently large to reduce the number of grains on the considered time scales. This is why the application of a stronger electric field only improves the alignment in the field direction ( $z$ axis), but has no effect on the grain structure in the $x-y$ plane [compare Figs. 1(a) and 1(b)]. This effect is also well pronounced in the Fourier transforms of the patterns, showing nearly homogeneous rings of the first and second order in Figs. 2(a) and 2(b). These scattering patterns support our conclusion based on the visual inspection, suggesting that there is no preferred in-plane orientation. 


\section{Shear rate}
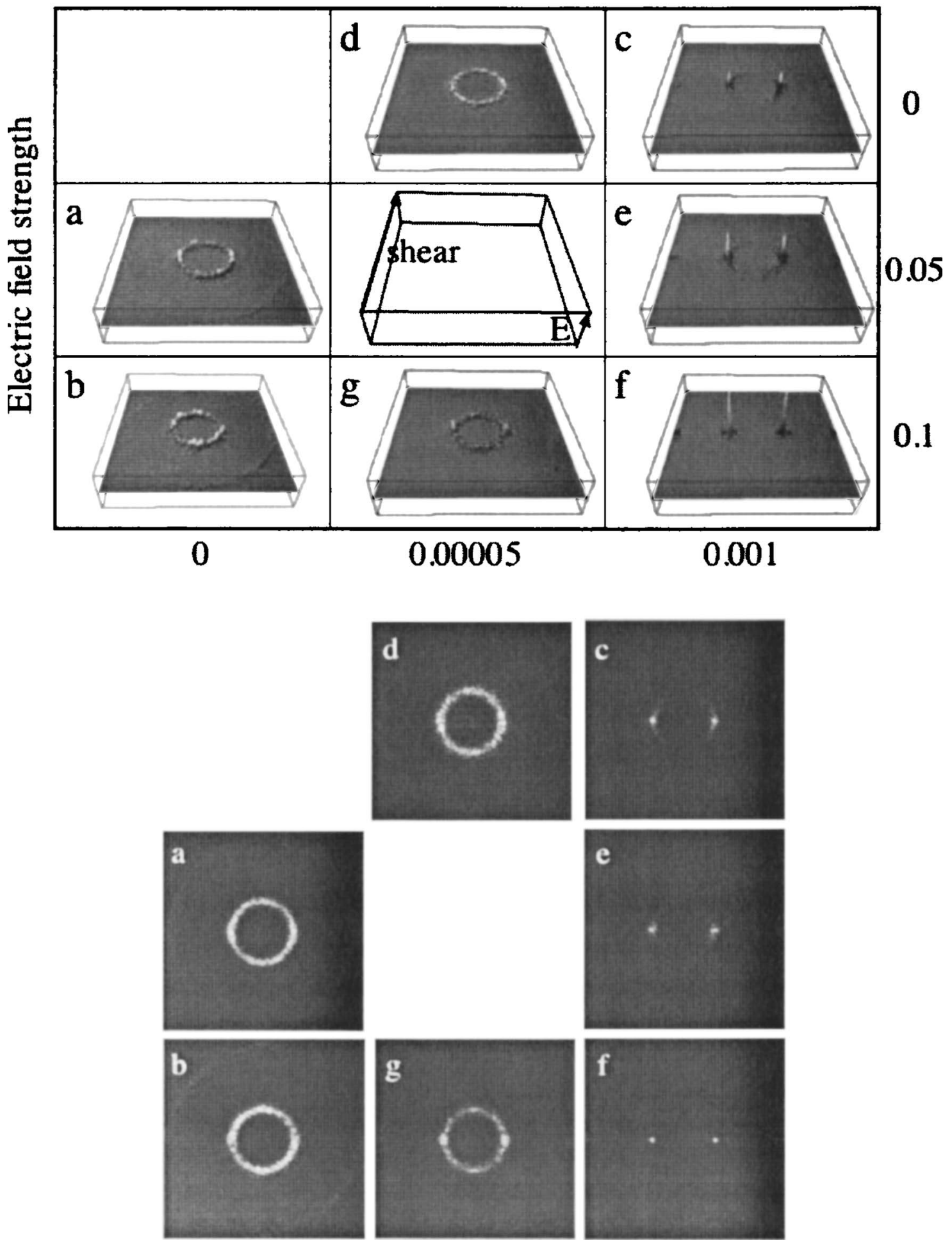

FIG. 2. Top: Two-dimensional scattering patterns ( $q x-q y$ slice at $q z=0$ of the 3D scattering volumes calculated by the fast Fourier transforms) for all simulated systems at time step 4000. The vertical axis shows the value of the intensity at given positions in the 2D slice. Bottom: Same as top, but now the intensities are given by a coloring scheme: white, high intensity: black, low intensity.

Rather than a single direction, simple shear has a preferred plane, namely, the shear plane. Therefore, it would - in principle - be able to form a single grain. Figures $1(\mathrm{c})$ and $1(\mathrm{~d})$ show snapshots of the simulation of the diblock copolymer melt after the application of shear flow only. We investigated two very different values of the shear rate. At a higher shear rate, the system mostly forms the so-called perpendicular lamellae, ${ }^{14,24}$ similar to earlier results. ${ }^{20}$ This is well seen both from visual inspection of the structure shown in Fig. 1(c) and the presence of two peaks in the Fourier transform [Fig. 2(c)]. The system is still rich of defects in the shear gradient-vorticity plane $(z-y$ plane), Fig. 1(c). In the 
reciprocal $q z-q y$ plane we observe even broader peaks (not shown here) when compared to the ones in Fig. 2(c) (representing the $q x-q y$ plane). Although the shear has two preferred directions (flow and shear gradient), they are not equivalent in their action on the alignment of the microstructure. Shear forces in the flow direction will obviously destroy all structures with wave vectors in the flow direction, leading to an alignment of the lamellae along the flow direction, as already seen in Fig. 1(c). The formation of a single grain in the shear gradient-vorticity plane occurs due to the combination of two effects. One is the energy cost of grain boundaries, which is the same as in the case of the electric field only, Figs. 1(a) and 1(b). The same arguments as before therefore hold, and the associated force is rather low. Another effect is due to the preferred orientation of a sheared lamellar system in the gradient-vorticity plane. ${ }^{14}$ The latter effect is associated with a small parameter (intrinsic for the material) responsible for the angle coupling. ${ }^{24}$ It is small as well. A much lower shear rate value produces a structure [Fig. 1(d)] with a noticeable alignment in the $x$ direction (shear direction), but many differently oriented clusters in the $z-y$ plane. The Fourier transform of this structure [Fig. $2(d)]$ supports this observation by showing a ring with two very shallow peaks along the $q y$ axis. The Fourier transforms in both the $q x-q z(q y=0)$ and $q y-q z(q x=0)$ planes are very similar to the ring shown in Fig. 2(d), with shallow peaks along the $q z$ axis (figure not shown here). From the theory in Ref. 24 it is known that low shear rates produce the so-called parallel lamellae, with the lamellae being parallel to the shear plane. From a simulation point of view these systems are hard to simulate, as they require very long shearing (and subsequently, enormous computational effort) to get any reasonable and noticeable alignment. Therefore, we limit ourselves to the result in Fig. 1(d), where alignment in the shear flow direction is already present in both the real and reciprocal space images, but, as a whole, the lamellae are a mixture of perpendicular and parallel clusters [reflected by the dark patches in Fig. 1(d)].

Combining the two external fields in an orthogonal way can lead to a defect-free microstructure. The results in Figs. 1(a)-1(d) suggest that the best result will be achieved if the electric field will be chosen normal to the shear plane. This suggestion is confirmed in Figs. 1(e)-1(g). At a high shear rate the applied flow field promotes perpendicular lamellae with some remaining defects in the $z$ direction; the electric field applied in the $z$ direction eliminates these defects. The higher the electric field the better the overall alignment of the final structure is [one should compare Figs. 1(e) and 1(f)]. For lower shear rates we observe a preferred orientation [two peaks in the Fourier transform in Fig. 2(g)] but also many grains with various orientations in the $x-y$ plane, reflected in the presence of a ring in the reciprocal $q x-q y$ plane. Nevertheless, application of a shear field with a lower shear rate, in combination with an applied electric field, gives rise to a better overall structural orientation than the case of either of the fields alone, which can be clearly seen from the more pronounced peaks in Fig. 2(g) compared to the ones in Fig. 2(d) (only shear) and the absence of peaks in Fig. 2(b) (only electric field). However, the structure is clearly not as perfect as in Figs. 1(e) and 1(f), where the applied shear field is stronger. For this relatively high shear rate the peaks in Fourier transform are very sharp. No visible ring is present, Figs. 2(e) and 2(f), indicating an overall tendency for the lamellae to orient in the preferred (shear gradient) direction. With increasing electric-field strengths [Figs. 2(c), 2(e) and 2(f)], secondary peaks in the $q y$ direction are becoming more and more prominent. The best structure in terms of defect-free alignment on a larger scale is achieved in Fig. 2(f), for a combination of high shear rate and high electric-field strength.

The simulation findings are well in line with the recent experimental findings by $\mathrm{Xu}$ et al. ${ }^{10}$ that three-dimensional control over lamellar microdomain orientation can be achieved by using two orthogonal fields. The setup of our simulation is a bit different from these experiments, ${ }^{10}$ where an elongational flow and an electric field have been applied to the sample sequentially. We have chosen a simpler physical model, which, however, contains the necessary components to explain the effect of orthogonal flow and electric field. One more difference is that the flow setup in Ref. 10 produced oriented nuclei of parallel lamellae, similar to our Fig. 1(d), although the final and desired orientation was perpendicular. The experimental transmission electron microscopy (TEM) images show that some defects still remain in the sample even after the application of the two orthogonal fields. ${ }^{10}$ Our results suggest that preshearing (for example, by oscillatory shear ${ }^{14}$ ) with a shear strength that promotes perpendicular lamellae $e^{20,24}$ will lead to an accelerated reorientation and an even less-defected structure at the end.

\section{CONCLUSIONS}

Large-scale computer simulations have shown that the orientation of lamellar microdomains can be controlled in three dimensions by the use of two orthogonal external fields. A shear field was applied to a diblock copolymer melt to control the orientation in the flow direction and an external electric field was applied to control the orientation normal to the shear plane, resulting in an excellent long-range order and orientation of the lamellar microdomains. The results present a theoretical explanation of the phenomena observed in recent experiments. ${ }^{10}$ We suggest that preshearing, which would promote a perpendicular rather than a parallel orientation, would lead to an even less-defected final microstructure.

After the present work was finished, we learned about the work by Feng and Ruckenstein. ${ }^{25}$ In their excellent study they combined the effect of shear, electric field, and selective surfaces. They concentrated on the hexagonal cylindrical and lamellar systems, and used a Landau-Ginzburg Hamiltonian. Their dynamics was incorporated by a cell dynamics simulation scheme based on Cahn-Hilliard theory. The treatment of the electric field was adapted from our earlier papers.

The results of both studies support each other. One can compare Figs. 5(b), 7, and 20 in Ref. 25 with the results in the present work, which both demonstrate the formation of the perfect $3 \mathrm{D}$ ordering in a lamellar system with two orthogonal fields applied. However, we remark that our present 
study has an additional value. As was explained by Fredrickson in Ref. 24, Landau-Ginzburg Hamiltonian with constant coefficients does not distinguish between parallel and perpendicular lamellar orientations in shear flow. That means that any lamellar orientation with their normal perpendicular to the flow direction can be seen in a simulation based on Landau-Ginzburg Hamiltonian. Indeed, Figs. 3(b) and 16(e) in Ref. 25 show simulation boxes with several lamellar domains of different orientations, which resemble a set of parallel and perpendicular lamellae (some bended). However, the work in Ref. 25 uses only a single value for the shear rate, and our statement would be more rigorous if we had known their results for different values of the shear rate. This can be a subject for future work. Contrary to their treatment, the angle dependency of the coefficients in Landau-Ginzburg Hamiltonian is automatically taken into account by the selfconsistent theory employed in our study. This theory does not use Landau-Ginzburg Hamiltonian in calculations, but such Hamiltonian can be obtained as a limiting case of the self-consistent field theory in weak segregation. As the important question is about controlling the $3 \mathrm{D}$ orientation, all effects should be systematically considered. Therefore, the present paper and the work of Ref. 25 complement each other and both clarify the physical picture behind the phenomenon.

\section{ACKNOWLEDGMENT}

Supercomputer time was provided by NCF (Stichting Nationale Computerfaciliteiten).

\footnotetext{
${ }^{1}$ T. Thurn-Albrecht, J. Schotter, G. A. Kastle et al., Science 290, 2126 (2000).

${ }^{2}$ Y. Cohen, R. J. Albalak, B. J. Dair, M. S. Capel, and E. L. Thomas,
} Macromolecules 33, 6502 (2000).
${ }^{3}$ C. C. Honeker and E. L. Thomas, Macromolecules 33, 9407 (2000).

${ }^{4}$ A. Keller, E. Pedemonte, and F. M. Willmouth, Nature (London) 225, 538 (1970).

${ }^{5}$ Y. Kobori, Y. K. Kwon, M. Okamoto, and T. Kotaka, Macromolecules 36, 1656 (2003).

${ }^{6}$ K. Amundson, E. Helfand, D. D. Davis, X. Quan, S. S. Patel, and S. D. Smith, Macromolecules 24, 6546 (1991).

${ }^{7}$ T. Morkved, M. Lu, A. M. Urbas, E. E. Ehrichs, H. M. Jaeger, P. Mansky, and T. P. Russell, Science 273, 931 (1996).

${ }^{8}$ E. Huang, L. Rockford, T. P. Russell, C. J. Hawker, and J. Mays, Nature (London) 395, 757 (1998).

${ }^{9}$ M. Kimura, M. J. Misner, T. Xu, S. H. Kim, and T. P. Russell, Langmuir 19, 9910 (2003).

${ }^{10}$ T. Xu, J. T. Goldbach, and T. P. Russell, Macromolecules 36, 7296 (2003).

${ }^{11}$ A. V. Zvelindovsky and G. J. A. Sevink, Phys. Rev. Lett. 90, 049601 (2003).

${ }^{12}$ R. J. Albalak and E. L. Thomas, J. Polym. Sci., Part B: Polym. Phys. 31, 37 (1994).

${ }^{13}$ R. J. Albalak and E. L. Thomas, J. Polym. Sci., Part B: Polym. Phys. 32, 341 (1993).

${ }^{14}$ U. Wiesner, Macromol. Chem. Phys. 198, 3319 (1997).

${ }^{15}$ T. Thurn-Albrecht, J. DeRouchey, T. P. Russell, and R. Kolb, Macromolecules 35, 8106 (2002).

${ }^{16}$ A. Boker, A. Knoll, H. Elbs, V. Abetz, A. H. E. Muller, and G. Krausch, Macromolecules 35, 1319 (2002).

${ }^{17}$ A. Onuki and J. Fukuda, Macromolecules 28, 8788 (1995).

${ }^{18}$ B. A. C. van Vlimmeren, N. M. Maurits, A. V. Zvelindovsky, G. J. A. Sevink, and J. G. E. M. Fraaije, Macromolecules 32, 646 (1999).

${ }^{19}$ A. Onuki, Phase Transition Dynamics (Cambridge University Press, Cambridge, 2002)

${ }^{20}$ A. V. Zvelindovsky, G. J. A. Sevink, B. A. C. van Vlimmeren, N. M. Maurits, and J. G. E. M. Fraaije, Phys. Rev. E 57, R4879 (1998).

${ }^{21}$ L. D. Landau and E. M. Lifshitz, Electrodynamics of Continuous Media (Pergamon, Oxford, 1960), Chap. II.

${ }^{22}$ A. Böker, H. Elbs, H. Hänsel et al., Macromolecules 36, 8078 (2003).

${ }^{23}$ K. Amundson, E. Helfand, X. Quan, S. D. Hudson, and S. D. Smith, Macromolecules 27, 6559 (1994).

${ }^{24}$ G. H. Fredrickson, J. Rheol. 38, 1045 (1994).

${ }^{25}$ J. Feng and E. Ruckenstein, J. Chem. Phys. 121, 1609 (2004). 